\title{
Vessel Wall Thickening and Enhancement in High-Resolution Intracranial Vessel Wall Imaging: A Predictor of Future Ischemic Events in Moyamoya Disease
}

\author{
(D) A. Kathuveetil, (DP.N. Sylaja, (D). Senthilvelan, (D) K. Chandrasekharan, (D) M. Banerjee, and (D) B. Jayanand Sudhir
}

\section{ABSTRACT}

BACKGROUND AND PURPOSE: Very few data are available with regard to high-resolution intracranial vessel wall imaging characteristics of Moyamoya disease and their relation to ischemic stroke risk. We investigated the high resolution imaging characteristics of MMD and its correlation with recent ischemic events.

\begin{abstract}
MATERIALS AND METHODS: Patients with Moyamoya disease confirmed by DSA, including patients after revascularization, were enrolled. All the patients underwent high-resolution intracranial vessel wall imaging. Vessel wall thickening, enhancement, and the remodeling index of the bilateral distal ICA and proximal MCA were noted. The patients were followed up at 3 months and 6 months after high-resolution intracranial vessel wall imaging and the association of ischemic events with imaging characteristics was assessed.
\end{abstract}

RESULTS: Twenty-nine patients with Moyamoya disease were enrolled. The median age at symptom onset was 12 years (range, 1-51 years). A total of 166 steno-occlusive lesions were detected by high-resolution intracranial vessel wall imaging. Eleven lesions with concentric wall thickening (6.6\%) were noted in 9 patients. Ten concentric contrast-enhancing lesions were observed in 8 patients, of which 3 patients (4 lesions) showed grade II enhancement. The presence of contrast enhancement $(P=.01)$ and wall thickening $(P \leq .001)$ showed a statistically significant association with ischemic events within 3 months before and after the vessel wall imaging. Grade II enhancement showed a statistically significant $(P=.02)$ association with ischemic events within 4 weeks of high-resolution intracranial vessel wall imaging. The mean \pm standard deviation outer diameter of the distal ICA (right, $-3.3 \pm$ $0.68 \mathrm{~mm}$; left, $3.4 \pm 0.60 \mathrm{~mm}$ ) and the remodeling index (right, $0.71 \pm 0.13$; left, $0.69 \pm 0.13$ ) were lower in Moyamoya disease.

CONCLUSIONS: High-resolution intracranial vessel wall imaging characteristics of concentric wall thickening and enhancement are relatively rare in our cohort of patients with Moyamoya disease. The presence of wall thickening and enhancement may predict future ischemic events in patients with Moyamoya disease.

ABBREVIATIONS: $\mathrm{ACA}=$ anterior cerebral artery; HRVWI = high-resolution intracranial vessel wall imaging; $\mathrm{MMD}=$ Moyamoya disease; $\mathrm{PCA}=$ posterior cerebral artery; SD = standard deviation

M oyamoya disease (MMD) is a rare cerebrovascular disease characterized by progressive steno-occlusive changes at the terminal portion of the ICA associated with the development of a fine basal collateral network. ${ }^{1-3}$ In the routine clinical practice,

Received September 12, 2019; accepted after revision October 27.

From the Comprehensive Stroke Care Program (A.K., P.N.S.), Department of Neurology; Departments of Radiology (S.S., K.C.) and Neurosurgery (B.J.S.), Rajiv Gandhi Centre for Biotechnology, Trivandrum, Kerala, India; and Sree Chitra Tirunal Institute for Medical Sciences and Technology (M.B.), Trivandrum, Kerala, India.

This study was funded by Wellcome Trust-Department of Biotechnology (DBT)/ India Alliance as part of Research Training Fellowship.

Please address correspondence to P.N. Sylaja, MD, DM, Neurology Comprehensive Stroke, Care Program, Sree Chitra Tirunal Institute for Medical Sciences and

Technology, Trivandrum, India 695011; e-mail: sylajapn@hotmail.com; @DrArunKNair

- Indicates open access to non-subscribers at www.ajnr.org

http://dx.doi.org/10.3174/ajnr.A6360 particularly in the Asian population where both intracranial atherosclerotic disease and MMD are common and may present with similar angiographic features, clinicians face difficulties in differentiating them, which may delay the appropriate management. High-resolution intracranial vessel wall imaging (HRVWI) has recently been developed as a reliable tool to distinguish MMD from other intracranial vessel pathologies. ${ }^{2,4,5}$ There are few studies that describe concentric vessel wall enhancement that involves the distal ICA and proximal MCA in patients with symptomatic and patients with asymptomatic MMD. ${ }^{1,4}$ On the symptomatic side, concentric enhancement was observed in $82.1 \%$ of distal ICAs and $94.1 \%$ of proximal MCAs. ${ }^{1}$ Also, concentric enhancement of bilateral distal ICAs was observed exclusively in MMD compared with intracranial atherosclerotic disease (53\% versus $0 \%) .{ }^{1}$ In addition, one recent study also observed a significant 
association of vessel wall enhancement with cerebral ischemic events in patients with MMD, which suggests the role of inflammation and neovascularization. ${ }^{3,6,7}$ Little is known about the characteristic HRVWI findings and the clinical significance of those findings in MMD. This study sought to investigate the high-resolution imaging characteristics of MMD and their correlation with recent ischemic events in an Indian population.

\section{MATERIALS AND METHODS \\ Patient Data}

Patients with MMD who satisfied the diagnostic criteria were included in the study. ${ }^{8}$ The inclusion criteria were the following: 1) patients with $\mathrm{MMD}$, including postrevascularization cases and those medically stable enough to be in the MR scanner for 30 to 90 minutes without general anesthesia and/or sedation, and 2) good quality images. The exclusion criteria were as follows: 1) potential sources of cardioaortic embolism, 2) $\geq 50 \%$ extracranial stenosis, 3) vasculopathies, such as dissection, vasculitis, or Takayasu arteritis, 4) failure of renal function (glomerular filtration rate $<60 \mathrm{~mL} / \mathrm{min}$ ), or 5) contraindications to MR imaging. The criteria for selecting a patient for cerebral revascularization included 1) cerebral infarction or hemorrhage based on the patient's functional disability and 2) Suzuki stage II to IV in angiographic staging. The timing of the operation was at least 4-6 weeks in patients with stroke.

Surgery for a patient who is asymptomatic or has an asymptomatic hemisphere is rarely offered. All the patients provided informed consent, and the study was approved by the institutional ethics committee. The clinical features and demographics of the patients were collected, and the images were reviewed. After HRVWI, the patients were followed up at 3 months and 6 months. They were assessed clinically for new-onset strokes, TIA, and headaches, and for syncope. The association of acute ischemic events with HRVWI characteristics was noted. HRVWI characteristics of the patients who underwent surgical revascularization were noted and the association of imaging findings with ischemic events was analyzed.

\section{HRVWI Protocol}

The included subjects underwent pre- and postcontrast HRVWI after obtaining informed consent. HRVWI was performed as per previously published protocol from Sree Chitra Tirunal Institute for Medical sciences and technology, Trivandrum, India on a 3T Discovery system (GE Healthcare, Milwaukee, Wisconsin). ${ }^{9}$ Acquired sequences (corresponding voxel resolution before interpolation, after interpolation) are shown as follows: sagittal Cube (GE Healthcare) T1 $(0.8 \times 0.8 \times 1.4 \mathrm{~mm}, 0.3 \times 0.3 \times 1.4 \mathrm{~mm})$, sagittal Cube T2 $(0.6 \times 0.6 \times 1.2 \mathrm{~mm}, 0.3 \times 0.3 \times 1.2 \mathrm{~mm})$, sagittal Cube proton density $(0.6 \times 0.5 \times 1.2 \mathrm{~mm}, 0.3 \times 0.3 \times$ $1.2 \mathrm{~mm}$ ). Interpolation was done by using zero-filling interpolation X 512 (matrix or in-plane) zero-filling interpolation X 2 (section or through-plane). Also, SWI and DWI sequences were also acquired. These sequences eventually resulted in the following scan time for each sequence: sagittal CUBE T1 fat suppressed, 6:49 minutes; sagittal Cube T2, 6:04 minutes; sagittal Cube proton density, 4:28 minutes; and postcontrast sagittal Cube T1 fat suppressed, 6:49 minutes. Postcontrast images were acquired after
30 seconds of intravenous administration of gadoterate meglumine (Dotarem; Guerbet, Aulnay-sous-Bois, France) Guerbet LLC, headquarters located in Princeton, US at a dose of 0.1$\mathrm{mmol} / \mathrm{kg}$ body weight.

\section{Image Review}

The conventional MR imaging, DSA-Digital subtraction angiography, and HRVWI were read by 1 neuroradiologist S. Senthilvelan and 1 neurologist K. Arun with 4 years of experience and were blinded to the clinical information. All the images were read in consensus. MR images were reviewed for the presence of acute and chronic infarcts, hemorrhages, the pattern of infarcts, and the presence of small-vessel ischemic changes. All patients underwent DSA by using a biplane angiography system (Philips Healtcare, Best, the Netherlands) in an interventional radiology angiography suite. Luminal images were evaluated for the presence of stenosis and/or occlusion that involved distal ICA, proximal MCA, anterior cerebral artery (ACA), posterior cerebral artery (PCA), and basilar artery, and for the type of collaterals. Collaterals were classified as superficial meningeal (leptomeningeal, durocortical) and deep parenchymal (subependymal anastomotic, inner thalamic) networks. ${ }^{10}$ The disease was staged in all the patients by using DSA based on the Suzuki staging: 1) narrowing of carotid bifurcation, 2) dilation of the ACA and MCA with initiation of Moyamoya, 3) partial disappearance of the ACA and MCA with intensification of ICA Moyamoya, 4) advanced steno-occlusive changes in the ICA with a small amount of ICA Moyamoya, 5) absence of the ACA and MCA with further reduction of ICA Moyamoya, and 6) complete disappearance of ICA Moyamoya. ${ }^{11}$

HRVWI was reviewed for wall thickening and wall enhancement that involved the distal ICA, proximal MCA, ACA, PCA, and basilar artery. The remodeling index of only the distal ICA was assessed considering the large caliber and early involvement of distal ICA in MMD:

Wall Thickening. Wall thickening was classified as concentric, eccentric, or no wall thickening by using a precontrast T1 Cube sequence by visual inspection. Concentric wall thickening was defined as a circumferential and uniform thickening, with the width of the thinnest wall segment $\geq 50 \%$ of the thickest segment. Eccentric wall thickening was defined as either limited to 1 side of the vessel wall, or, where circumferential enhancement was noted, the thinnest part of the wall enhancement was estimated to be $<50 \%$ of the thickest point. ${ }^{12}$

Contrast Enhancement. The degree of enhancement of vessels was qualitatively graded, depending on its signal intensity on postcontrast T1 images compared with corresponding precontrast images: grade 0, no enhancement; grade I, mild enhancement, the signal intensity of vessel wall is less than that of the pituitary infundibulum; and grade II, strong enhancement, the signal intensity of contrast-enhanced vessel wall is similar to or greater than that of the infundibulum. ${ }^{3,13}$ Enhancement was considered concentric if it was circumferential and uniform (defined specifically as the width of the thinnest wall segment of $\geq 50 \%$ of the thickest segment). Eccentric enhancement was defined as 
Table 1: Demographic and clinical characteristics ( $N=29)$

\begin{tabular}{lc}
\hline \multicolumn{1}{c}{ Patient Characteristics } & Results \\
\hline Sex, $n(\%)$ & $17(58.6)$ \\
$\quad$ Males & $12(41.6)$ \\
Females & \\
Age, $n(\%)$ & $15(51.7)$ \\
$<18$ y & $14(48.3)$ \\
$\geq 18$ y & $-12(6-36)$ \\
Age at first event ${ }^{a}$, median (IQR), y & \\
Initial clinical presentation, $n(\%)$ & $10(34.5)$ \\
Ischemic stroke & $5(17.2)$ \\
Intracerebral bleed & $7(24.2)$ \\
TIA & $1(3.4)$ \\
Syncope & $6(20.7)$ \\
Seizure & $22(75.9)$ \\
Recurrent events, $n$ (\%) & $10(34.5)$ \\
Ischemic stroke & $2(6.9)$ \\
Intracranial bleed (intraventricular hemorrhage) & $2(6.9)$ \\
Positive family history of MMD, $n(\%)$ & $1(0-3)$ \\
NIHSS on admission, median (IQR) & $1(0-3)$ \\
mRS on admission, median (IQR)
\end{tabular}

Note:-IQR indicates interquartile range; $\mathrm{mRS}$, modified Rankin scale.

${ }^{a}$ The mean \pm SD interval between the first symptom and HRVWI was $64.7 \pm$ 80.7 months. either limited to 1 side of the vessel wall (eg, not $360^{\circ}$ circumferential enhancement) or, where circumferential enhancement was noted, the thinnest part of the wall enhancement was estimated to be $<50 \%$ of the thickest point. ${ }^{1,12}$

Remodeling Index. The outer diameter of the terminal portion of the ICA was measured by using sagittal T1 fat-suppressed images. The remodeling index was calculated as the outer diameter of a narrowed vessel (measurements between both outer diameters) divided by the caliber of the normal proximal reference vessel ${ }^{1}$ (proximal ICA). If the ratio is $>1.05$, then it was taken as positive remodeling, $<0.95$ was negative remodeling, and $0.95-1.05$ was normal. ${ }^{14}$ The caliber of the vessel was calculated in the sagittal-oblique images perpendicular to the vessel in axial sections.

\section{Statistical Analysis}

Continuous-median (interquartile range) or mean standard deviation (SD) categorical variables-percentage (count), respectively. Data comparison of categoric variables was done by using the Fisher exact test. A $P$ value of $<.05$ was considered statistically significant. The data were analyzed by using analytical software SPSS Statistics for Windows, version 21.0 (2012) (IBM, Armonk, New York).

Table 2: Imaging characteristics of patients with MMD

\begin{tabular}{|c|c|c|}
\hline \multirow{2}{*}{$\begin{array}{lr} & \text { Characteristic } \\
\text { A. MRI }\end{array}$} & \multicolumn{2}{|c|}{$n(\%)$ ( $n=58$ Hemispheres) } \\
\hline & & \\
\hline 1. Pattern of infarct & Acute Infarcts & Chronic Infarcts \\
\hline Watershed & $9(15.5)$ & $15(25.9)$ \\
\hline Territorial & $3(5.2)$ & $5(8.6)$ \\
\hline Cortical & $2(3.4)$ & $3(5.2)$ \\
\hline Isolated subcortical & $1(1.7)$ & $2(3.4)$ \\
\hline \multicolumn{3}{|l|}{ B. DSA } \\
\hline \multicolumn{3}{|l|}{ 1. Laterality $(n=29)$} \\
\hline Bilateral & $27(93.1)$ & \\
\hline Unilateral & $2(6.9)$ & \\
\hline 2. Vessels involved ( $n=166$ vessels) & Narrowed & Occluded \\
\hline Distal ICA & 19 (11.4) & $37(22.2)$ \\
\hline Proximal MCA & $12(7.2)$ & $39(23.5)$ \\
\hline Proximal ACA & $17(10.2)$ & $25(15.0)$ \\
\hline PCA & $8(4.8)$ & $1(0.6)$ \\
\hline Basilar artery (29 vessels) & $1(0.6)$ & $0(0)$ \\
\hline 3. Suzuki stage & Right & Left \\
\hline I & $2(7.1)$ & $2(7.1)$ \\
\hline II & $0(0)$ & $1(3.6)$ \\
\hline III & $5(17.9)$ & $2(7.1)$ \\
\hline IV & $4(14.3)$ & $10(35.7)$ \\
\hline V & $16(57.1)$ & $13(46.4)$ \\
\hline VI & $1(3.6)$ & $0(0)$ \\
\hline \multicolumn{3}{|l|}{ 4. Collaterals ( $n=58$ hemispheres) } \\
\hline \multicolumn{3}{|l|}{ Superficial meningeal } \\
\hline \multicolumn{3}{|l|}{ Leptomeningeal } \\
\hline Anterior & $27(46.6)$ & $24(41.4)$ \\
\hline Posterior & $21(36.2)$ & $20(34.5)$ \\
\hline \multicolumn{3}{|l|}{ Durocortical } \\
\hline Anterior & $21(36.2)$ & $18(31.1)$ \\
\hline Posterior & $3(5.2)$ & $5(8.6)$ \\
\hline \multicolumn{3}{|l|}{ Deep parenchymal } \\
\hline \multicolumn{3}{|l|}{ Subependymal anastomotic } \\
\hline Anterior & $6(10.3)$ & $6(10.3)$ \\
\hline Posterior & $5(8.6)$ & $5(8.6)$ \\
\hline \multicolumn{3}{|l|}{ Inner thalamic } \\
\hline Anterior & $8(13.8)$ & $6(10.3)$ \\
\hline Posterior & $8(13.8)$ & 7 (12.1) \\
\hline
\end{tabular}

\section{RESULTS}

Twenty-nine patients with MMD, confirmed by DSA, were enrolled in the study. Of them, 15 patients had undergone surgical revascularization. Seventeen (58.6\%) were males and $12(41.4 \%)$ were females (male-to-female ratio, 1.4 ) and $51.7 \%$ were $<18$ years old. The median age at symptom onset was 12 years (range, 151 years), with ischemic stroke (34.5\%) being the most common initial clinical presentation, followed by TIA $(24.1 \%)$, seizures $(20.7 \%)$, intracranial hemorrhage (17.2\%), headache (6.8\%), and syncope (3.4\%). Seizures and hemorrhage as initial presentation occurred predominantly in children $<10$ years old $(P=.03)$ and in adults $>30$ years old $(P=.04)$, respectively. Recurrence of neurologic events was observed in $75.9 \%$ of the patients (Table 1 ). A total of 58 hemispheres, including 2 cases of unilateral MMD, were analyzed by using MR imaging, which showed 9 watershed, 3 territorial, 2 cortical, and 1 subcortical infarct in the MCA territory. DSA showed predominantly occlusive lesions that involved the distal ICA, proximal MCA, ACA, and PCA (Table 2) and collaterals, predominantly originating from the superficial meningeal network, which was reinforced by the deep parenchymal network (Table 2).

HRVWI was performed in 29 patients. A total of 166 steno-occlusive lesions were 
detected (56 distal ICA, 51 proximal MCA, 49 proximal ACA, 9 PCA, and 1 basilar artery). Among them, 11 lesions (6.6\%) with concentric wall thickening (Figure) were noted in 9 patients (31.03\%), of which 9 lesions (81.8\%) involved the distal ICAs and 2 proximal MCAs (18.2\%). Ten concentric contrast-enhancing lesions $(6.02 \%)$ were noted in 8 patients $(27.6 \%)$, of whom 3 patients (4 lesions) showed lesions with grade II enhancement. Grade I enhancement involved 4 distal ICAs and 2 proximal MCAs. Grade II enhancement (Figure) was observed in 3 distal ICAs and 1 proximal MCA on the symptomatic side. None of the

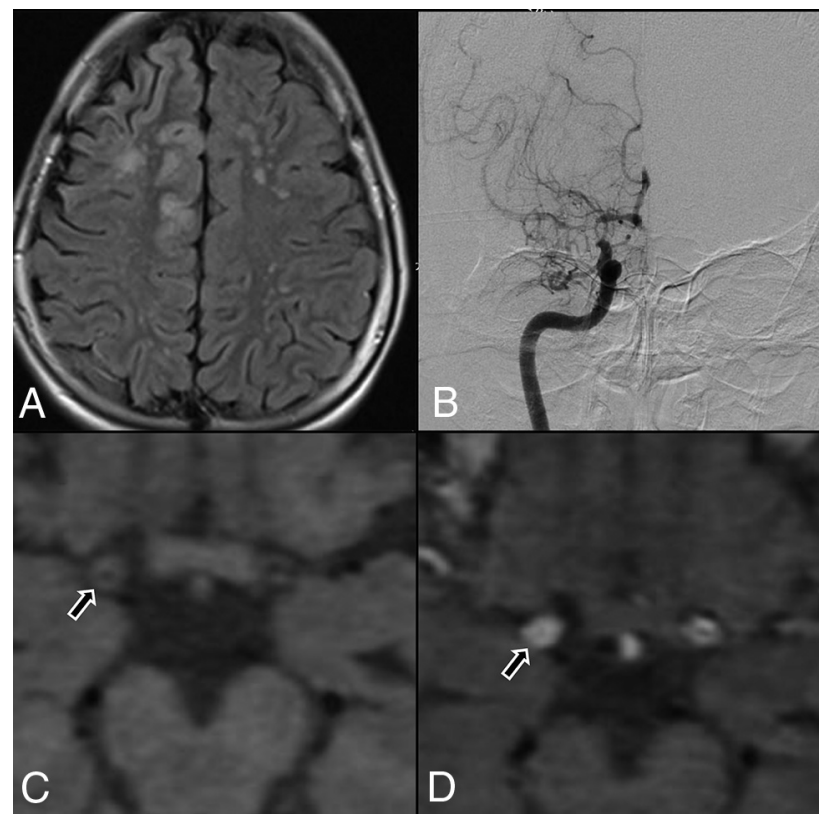

FIGURE. $A$, Flair axial section showing right ACA and MCA territory cortical infarct and multiple discrete MCA-ACA watershed infarct on the left side. $B$, DSA, showing tight stenosis of the distal ICA, with concomitant narrowing of proximal MCA and ACA, with leptomeningeal collaterals. C, Axial T1 noncontrast, HRVWI, showing circumferential wall thickening (arrow) and narrowing of the lumen of right distal ICA. D, Axial T1 contrast-enhanced, HRVWI, showing circumferential grade II enhancement (arrow) of a right distal ICA (Note:Enhancement is graded by comparing with pituitary infundibulum).
ACA, PCA, and basilar artery MMD lesions revealed wall thickening or enhancement.

The mean age at the initial presentation of patients with wall thickening and enhancement was 22.4 and 27.3 years, respectively. The mean \pm SD interval between the first symptom and HRVWI was $64.7 \pm 80.7$ months, which showed a statistically insignificant inverse relationship with $\mathrm{T} 1$ contrast enhancement (mean $\pm \mathrm{SD}, 55.6 \pm 49.6$ versus $68.1 \pm 90.6, P=.44$ ). The mean \pm SD follow-up period after the vessel wall imaging was $6.9 \pm 3.4$ months. The presence of contrast enhancement (50\%, $P=.01)$ and wall thickening $(64.3 \%, P \leq .001)$ showed a statistically significant association with the ischemic events within 3 months before and after vessel wall imaging. Grade II contrast enhancement showed a statistically significant $(P=.02)$ association with events within 4 weeks of HRVWI. The mean \pm SD outer diameter of the distal ICA (right, $3.3 \pm 0.68 \mathrm{~mm}$; left, $3.4 \pm 0.60 \mathrm{~mm}$ ) and mean $\pm \mathrm{SD}$ remodeling index (right, $0.71 \pm$ 0.13; left, $0.69 \pm 0.13$ ) were lower in MMD (Table 3).

Among the 15 patients (51.7\%) who underwent revascularization, $11(73.3 \%)$ underwent unilateral revascularization and 4 (26.7\%) underwent bilateral revascularization. HRVWI revealed contrast enhancement in only 1 patient who underwent bilateral revascularization and in 2 patients who underwent unilateral revascularization. Wall thickening was noted in 1 patient who underwent bilateral revascularization and 4 patients with unilateral revascularization. None of these patients developed ischemic events that corresponded to the arterial territory of wall thickening or enhancement during the follow-up period.

\section{DISCUSSION}

We analyzed the characteristic HRVWI findings in MMD and their possible role in predicting the risk of recent ischemic events. In our cohort of 29 patients with MMD, only 6.02\% MMD lesions showed contrast enhancement and 6.6\% MMD lesions showed wall thickening. Among them, wall thickening and enhancement were mostly observed in distal ICA and proximal MCA. The ACA, PCA, and basilar artery did not reveal such changes in HRVWI. A smaller outer diameter of the involved vessel with negative remodeling was also observed. Also, the

Table 3: HRVWI characteristics of MMD

\begin{tabular}{|c|c|c|}
\hline HRVWI Characteristics & $\begin{array}{l}\text { Contrast Enhancement } \\
\text { (8 Patients, } 10 \text { Lesions) }\end{array}$ & $\begin{array}{l}\text { T1 Wall Thickening } \\
\text { (9 Patients, } 11 \text { Lesions) }\end{array}$ \\
\hline \multicolumn{3}{|l|}{ Pattern, $n(\%)$} \\
\hline Concentric & $10(100)$ & $11(100)$ \\
\hline Eccentric & $0(0)$ & $0(0)$ \\
\hline \multicolumn{3}{|l|}{ Vessel involved, $n(\%)$} \\
\hline Distal ICA & $8(80)$ & $9(81.8)$ \\
\hline Proximal MCA & $2(20)$ & $2(18.2)$ \\
\hline Events within 3 mo before and after the imaging, $n(\%)$ & $7(87.5)(P=.01)$ & $9(100)(P \leq .001)$ \\
\hline Recent events (DWI positive) within 4 wk of imaging, $n(\%)$ & & $4(44.4)(P=.20)$ \\
\hline Grade I contrast enhancement & $0(0)$ & \\
\hline Grade II contrast enhancement & $3(100)(P=.02)$ & \\
\hline Suzuki stage & Grade 2 enhancement (4 lesions) & Wall thickening (11 lesions) \\
\hline I & $1(25)$ & $1(9.1)$ \\
\hline$\|$ & $1(25)$ & $2(18.2)$ \\
\hline III & 0 & $4(36.4)$ \\
\hline IV & $2(50)$ & $4(36.4)$ \\
\hline Remodeling index, mean \pm SD & Right, $0.71 \pm 0.13$ & Left, $0.69 \pm 0.13$ \\
\hline
\end{tabular}


presence of wall thickening and enhancement showed a statistically significant association with ischemic events.

We observed concentric wall enhancement in $27.6 \%$ of patients with MMD. Among the 166 steno-occlusive lesions detected by HRVWI, $6.02 \%$ of lesions only showed contrast enhancement, which is lower in comparison with the previously published data, which accounted for 70\%-93\%. ${ }^{1,3,15,16}$ This may be related to the characteristics of the population under study or might be related to the small sample size. There is evidence that MMD is primarily a proliferative disease of the intima and smooth muscles that leads to vascular stenosis and that concentric enhancement may represent hyperproliferation of vessel components. ${ }^{17}$ It is well known that strong contrast enhancement of the arterial wall suggests an increase in the vascular supply and increased endothelial permeability, which promotes entry of the contrast agent into the extravascular space. ${ }^{18}$

Chmelova et $\mathrm{al}^{19}$ found a higher expression of both angiogenetic factors in intima and vascular endothelial growth factor in the endothelium of Moyamoya-affected arteries than normal arteries. Nestin, a marker of newly formed endothelial cells was expressed in the Moyamoya-affected vessels. ${ }^{20}$ Thus, it can be presumed that an active angiogenetic milieu prevails in the enhanced vessels in patients with MMD. ${ }^{19}$ Similar to the atherosclerotic process in the extracranial vessels where concentric and circumferential wall enhancements suggest an inflammatory response due to increased macrophage infiltration and neovascularity, the enhancement in Moyamoya vessels may be considered to represent an active angiogenetic process and inflammatory response.

Vessel wall thickening was observed in $31.03 \%$ of the patients with MMD. Among the 166 steno-occlusive lesions detected, $6.6 \%$ of lesions only showed vessel wall thickening, which was diffuse and concentric. Also, the outer diameter of the distal ICA was attenuated with negative remodeling in all the patients. Histopathologic studies of ICAs and MCAs in MMD demonstrated eccentric intimal fibrous thickening, which is often multilayered, with newly formed elastic lamina between the layers. ${ }^{21}$ The internal elastic lamina is markedly tortuous, with duplication and a decrease in the number of smooth-muscle cells in the media and these pathologic changes are seen predominantly in the distal ICA, proximal MCA, and ACA. The thinning of media and decreased flow as well as constriction of the internal elastic lamina, culminate in the decreased outer diameter of the vessels as well as the negative remodeling. It can also be postulated that diffuse wall thickening might be due to vessel wall edema and investigators have found that the wall edema has a correlation with inflammation ${ }^{22}$ associated with significantly greater plasma concentration of matrix metalloprotease-9, monocyte chemoattractant protein-1, vascular endothelial growth factor, and other inflammatory cytokines.

Our study also observed steno-occlusive lesions, which predominantly involved the distal ICA, which supports the notion that stenosis in MMD begins first in the ICA and as the disease progress involves the MCA, followed by the ACA. The occlusive lesions in MMD predominantly involve the anterior circulation and the involvement of the vertebral and basilar arteries is relatively rare, ${ }^{21}$ which is also the same in our study. Our study could not demonstrate thickening or enhancement of the ACA, PCA, and basilar artery. However, in 1 study, $19.6 \%$ of ACAs showed contrast enhancement. ${ }^{3}$

We also studied the role of HRVWI to possibly predict a future event based on its characteristic findings. It was observed that grade II enhancing lesions were significantly associated with ischemic events within 4 weeks of imaging. Besides, those lesions that showed either wall thickening or contrast enhancement were significantly associated with neurologic events within 3 months before or after vessel wall imaging. Wang et $\mathrm{al}^{3}$ reported that high-grade enhancement in the Moyamoya-affected vessel is associated with an increased risk of acute ischemic infarction. As with atherosclerotic plaque, in which higher-grade contrast enhancement represents plaque instability and identifies lesions responsible for ischemic events, higher-grade enhancement in MMD may reflect a proliferative neovascular change and activity of the lesion. ${ }^{3}$ These findings suggest that HRVWI might help to identify patients at risk of an early event and help the physician triage them for early revascularization. We also observed that the presence of wall thickening and enhancement did not correlate with subsequent events after revascularization, which suggests that these findings cannot be taken into consideration in predicting the risk of future events after revascularization.

The strength of our study is that, because all the patients underwent detailed evaluation for secondary etiology, the study population truly represents idiopathic MMD. The HRVWI was read by a neuroradiologist and a neurologist who were blinded to clinical data and, hence, the imaging data are homogeneous and unbiased. Because all these patients were on a regular follow-up schedule, the recurrent cerebral events could be carefully documented. The study was conducted in a single center and the sample size was small. Also, the HRVWI was done at varying time intervals from the symptom onset with a long interval in a few of the patients, which may explain the relatively few patients with wall enhancement compared with previous studies. A study with a large sample size that involves multiple centers is needed to validate the findings in our study.

\section{CONCLUSIONS}

The presence of concentric wall thickening and enhancement in HRVWI was relatively rare in our cohort of patients with MMD compared with the Japanese and Chinese populations. The presence of wall thickening, and enhancement may predict future ischemic events in patients with MMD.

Disclosures: K. Arun-RELATED: Grant: Wellcome Trust/DBT*; Support for travel to meetings for the study or other purposes: Wellcome Trust/DBT.* P.N. Sylaja-RELATED: Grant: Wellcome DBT project* UNRELATED: Employment: Sree Chitra Tirunal Institute for Medical Sciences and Technology. C. KesavadasRELATED: Grant: Wellcome DBT* UNRELATED: Employment: Grants/Grants Pending: DST India. * ${ }^{*}$ Money paid to the institution.

\section{REFERENCES}

1. Ryoo S, Cha J, Kim SJ, et al. High-resolution magnetic resonance wall imaging findings of moyamoya disease. Stroke 2014;45:245760 CrossRef Medline

2. Han C, Li ML, Xu Y, et al. Adult moyamoya-atherosclerosis syndrome: clinical and vessel wall imaging features. J Neurol Sci 2016;369:181-84 CrossRef Medline 
3. Wang M, Yang Y, Zhou F, et al. The contrast enhancement of intracranial arterial wall on high-resolution MRI and its clinical relevance in patients with moyamoya vasculopathy. Sci Rep 2017;7:44264 CrossRef Medline

4. Kim YJ, Lee DH, Kwon JY, et al. High resolution MRI difference between moyamoya disease and intracranial atherosclerosis. Eur J Neurol 2013;20:1311-18 CrossRef Medline

5. Kesav P, Krishnavadana B, Kesavadas C, et al. Utility of intracranial high-resolution vessel wall magnetic resonance imaging in differentiating intracranial vasculopathic diseases causing ischemic stroke. Neuroradiology 2019;61:389-96 CrossRef

6. Hatsukami TS, Yuan C. MRI in the early identification and classification of high-risk atherosclerotic carotid plaques. Imaging Med 2010;2:63-75 CrossRef Medline

7. Wasserman BA. Advanced contrast-enhanced MRI for looking beyond the lumen to predict stroke: building a risk profile for carotid plaque. Stroke 2010;41(Suppl):S12-S16 CrossRef Medline

8. Fukui M. Guidelines for the diagnosis and treatment of spontaneous occlusion of the circle of Willis ('moyamoya' disease). Research Committee on Spontaneous Occlusion of the Circle of Willis (Moyamoya Disease) of the Ministry of Health and Welfare. Clin Neurol Neurosurg 1997;99:S238-40 CrossRef

9. Adhithyan R, Kesav P, Thomas B, et al. High-resolution magnetic resonance vessel wall imaging in cerebrovascular diseases. Neurol India 2018;66:1124-32 CrossRef Medline

10. Baltsavias G, Khan N, Valavanis A. The collateral circulation in pediatric moyamoya disease. Childs Nerv Syst 2015;31:389-98 CrossRef Medline

11. Zhao M, Zhang D, Wang S, et al. Posterior circulation involvement in pediatric and adult patients with moyamoya disease: a single center experience in 574 patients. Acta Neurol Belg 2018;118:227-33 CrossRef Medline

12. Swartz RH, Bhuta SS, Farb RI, et al. Intracranial arterial wall imaging using high-resolution 3-Tesla contrast-enhanced MRI. Neurology 2009;72:627-34 CrossRef Medline
13. Qiao $Y$, Steinman DA, Qin Q, et al. Intracranial arterial wall imaging using three-dimensional high isotropic resolution black blood MRI at 3.0 Tesla. J Magn Reson Imaging 2011;34:22-30 CrossRef Medline

14. Yu LB, Zhang Q, Shi ZY, et al. High-resolution magnetic resonance imaging of moyamoya disease. Chin Med J (Engl) 2015;128:3231-37 CrossRef Medline

15. Yuan M, Liu ZQ, Wang ZQ, et al. High-resolution MR imaging of the arterial wall in moyamoya disease. Neurosci Lett 2015;584:77-82 CrossRef Medline

16. Mossa-Basha $M$, de Havenon A, Becker KJ, et al. Added value of vessel wall magnetic resonance imaging in the differentiation of moyamoya vasculopathies in a non-Asian cohort. Stroke 2016;47:1782-88 CrossRef Medline

17. Fukui M, Kono S, Sueishi K, et al. Moyamoya disease. Neuropathology 2000;20(Suppl):S61-64 CrossRef Medline

18. Millon A, Boussel L, Brevet M, et al. Clinical and histological significance of gadolinium enhancement in carotid atherosclerotic plaque. Stroke 2012;43:3023-28 CrossRef Medline

19. Chmelova J, Kolar Z, Prochazka V, et al. Moyamoya disease is associated with endothelial activity detected by anti-nestin antibody. Biomed Pap Med Fac Univ Palacky Olomouc Czech Repub 2010;154:159-62 Medline

20. Mokrý J, Čížková D, Filip S, et al. Nestin expression by newly formed human blood vessels. Stem Cells Dev 2004;13:658-64 CrossRef Medline

21. Kaku Y, Morioka M, Ohmori Y, et al. Outer-diameter narrowing of the internal carotid and middle cerebral arteries in moyamoya disease detected on 3D constructive interference in steady-state MR image: is arterial constrictive remodeling a major pathogenesis? Acta Neurochir (Wien) 2012;154:2151-57 CrossRef Medline

22. Chen X, Zhao H, Chen Z, et al. Association between proximal internal carotid artery steno-occlusive disease and diffuse wall thickening in its petrous segment: a magnetic resonance vessel wall imaging study. Neuroradiology 2017;59:485-90 CrossRef Medline 